\section{Richtiges Verhalten bei Vernehmungen und sogenannten Qualitätssicherungsgesprächen}

In jüngster Zeit kommt es immer häufiger zu strafrechtlichen Ermittlungsverfahren in medizinischen Einrichtungen. In diesem Zusammenhang werden regelmäßig auch Notärzte und Notfallsanitäter/Rettungsassistenten angehört, sei es durch private Personen, etwa den Arbeitgeber, den ÄLRD bzw. Beauftragte des Klägers des ÄLRD, sei es unmittelbar durch die Ermittlungsbehörden selbst. Die nachfolgende Darstellung soll allen Betroffenen eine Handreichung über die rechtlichen Rahmenbedingungen und das richtige Verhalten in diesen oftmals kritischen Situationen bieten.

\section{Befragungen durch private Personen}

Sehr häufig kommt es vor bzw. parallel zu strafrechtlichen Ermittlungsverfahren auch zu Einladungen zu Gesprächen mit privaten Personen. Dies kann sowohl im Rahmen von sog. internen Ermittlungen der Fall sein, in welchen der Arbeitgeber selbst oder - was häufig gegeben sein wird - durch diesen beauftragte Rechtsanwälte bemüht sind, den Sachverhalt aufzuklären. Darüber hinaus kommt es aufgrund der besonderen Konstellation etwa im Bereich der medizinischen Notversorgung nicht selten zu Anhörungen durch dritte Personen, die nicht Arbeitgeber der zu vernehmenden Person sind, z. B. weil aufgrund von vertraglichen Regelungen bestimmte Leistungen, die der einen Körperschaft obliegen, durch eine andere Einrichtung übernommen werden (z. B. ÄLRD).

Je nach Verlauf einer solchen Anhörung hat die vernommene Person mit arbeitsrechtlichen, aber insbesondere auch strafrechtlichen Konsequenzen zu rechnen. Da dies schwerwiegende Folgen nach sich ziehen kann, ist grundsätzlich anzuraten, einen in der Thematik versierten Rechtsanwalt zu konsultieren, sobald eine Einladung zu einer arbeitsrechtlichen Anhörung ausgesprochen wird.

\section{Rechtliche Rahmenbedingungen}

Die Fragen, die sich im Zusammenhang mit solchen Vernehmungen durch private Personen stellen, liegen auf der Hand: Muss der Beschäftigte zu einem solchen Gespräch überhaupt erscheinen? Muss er darin Angaben machen? Und schließlich: Muss der Beschäftigte sich im Rahmen selbst belasten? Hier ist zwischen den beiden eingangs geschilderten Konstellationen strikt zu trennen.

Befragung durch den Arbeitgeber oder von diesem beauftragte Dritte

Die interne Ermittlung, das heißt die Aufklärung eines möglicherweise strafrechtlich relevanten Sachverhalts durch das davon berührte Unternehmen selbst, hat in Deutschland zwar noch keine lange Tradition, ist heute aber nicht mehr wegzudenken. Dass hierbei neben der Auswertung von Dokumenten und E-Mails auch die Vernehmung von Mitarbeitern des Unternehmens eine zentrale Rolle spielt, ist offensichtlich. Umso erstaunlicher ist es, dass der rechtliche Rahmen für derartige Gespräche bislang immer noch nicht durch ein - möglicherweise kommendes - Gesetz über interne Ermittlungen klar definiert ist, sondern vielmehr ausschließlich auf allgemeine Regelungen des Arbeitsrechts zurückgegriffen wird.

Nach diesen gilt für den Beschäftigten, dass er gegenüber seinem Arbeitgeber umfassend zur Auskunft verpflichtet ist. Nach ganz herrschender Ansicht ergibt sich diese Auskunftsverpflichtung aus $\S \S 666,675$ BGB und umfasst auch Auskünfte, die Selbstbelastendes zum Inhalt haben. Dies gilt jedenfalls dann uneingeschränkt, wenn das dem Beschäftigten zur Last gelegte Fehlverhalten den Kernbereich seines Arbeitsverhältnisses betrifft (was bei Befragungen im Zusammenhang mit Notarzt- und Rettungsdiensteinsätzen regelmäßig der Fall sein dürfte). Betrifft das relevante Verhalten diesen nicht unmittelbar, wird eine Abwägung unter einer solchen Befragung eventuell sogar
Verhältnismäßigkeitsaspekten zu erfolgen haben, die dann regelmäßig zugunsten des Beschäftigten ausgehen wird.

Hinsichtlich der eingangs aufgeworfenen Fragen gilt somit: Der Arbeitnehmer muss einer Einladung zu einem solchen Gespräch Folge leisten, er muss wahrheitsgemäße Angaben machen und er ist verpflichtet sich selbst zu belasten, wenn ihm Straftaten zu Last gelegt werden, die den Kernbereich seiner Tätigkeit betreffen.

Umstritten ist in der Literatur damit nur noch, wie eine Verwertung der in der arbeitsrechtlichen Anhörung gewonnenen Erkenntnisse durch die strafrechtlichen Ermittlungsbehörden erfolgen kann. Und hierbei erweist sich alle Diskussion als l'Art pour l'Art, denn in der Praxis ist festzustellen, dass derartige Erkenntnisse durch die Staatsanwaltschaften bislang nahezu uneingeschränkt verwertet werden.

Vor diesem Hintergrund lässt sich festhalten, dass der Arbeitnehmer im Einzelfall abzuwägen haben wird, ob er sich der arbeitsrechtlichen Befragung stellt und ob er darin tatsächlich vollständige Angaben macht oder ob er nicht vielmehr bereit ist, sich dem Risiko arbeitsrechtlicher Konsequenzen auszusetzen.

\section{WEITERLESEN?}

In „Fachwissen extra - das online +“ stellen wir Ihnen Übersichtsartikel aus weiteren Thieme-Zeitschriften vor, die von rettungsdienstlichem Interesse sind. Hier drucken wir Passagen ab, die das Thema in Kurzform anreißen. Unter https://eref.thieme.de/NDLMW oder über den QR-Code kommen Sie direkt zum ungekürzten Artikel.

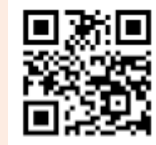

